(C) 2021 O. S. KUMPANENKO, H. Yu. HONCHAR, V. A. GOROBCHYSHYN, Yu. V. PROTSENKO

\title{
PRELIMINARY LIST OF SOME ACULEATA (HYMENOPTERA: CHRYSIDOIDEA, POMPILOIDEA, VESPOIDEA, APOIDEA) OF THE SHATSK NATIONAL NATURAL PARK (VOLYN REGION, UKRAINE)
}

Кумпаненко, О. С., Гончар, Г. Ю., Гороб̆чшин, В. А., Проценко, Ю. В. Попередній список деяких Aculeata (Нуmеnорtera: Chrysidoidea, Pompiloidea, Vespoidea, Ароidea) Шацького національного природного парку (Волинська область, Украӥна). Вісті Харківського ентомологічного товариства. 2021. Т. ХXIX, вип. 1. С. 8-19. DOI: 10.36016/KhESG-2021-29-1-2.

Наводиться попередній список Chrysididae, Mutillidae, Pompilidae, Vespidae, Sphecidae Crabronidae та Ароіdea Шацького національного природного парку. Серед 139 зареєстрованих на цей час 56 видів уперше вказуються для території парка, 5 видів внесено до Червоних книг України, Білорусі та Польщі, а 83 види належать до охоронних категорій МСОП. Один інвазійний вид (Isodontia mexicana) вперше знайдений у Волинській області.

Ключові слова: список видів, охоронні категорії, риючі оси, дорожні оси, бджоли. 1 рис., 14 назв.

Кумпаненко, А. С., Гончар, А. Ю., Г Горобчишин, В. А., Проценко, Ю. В. Предварительный список некоторых Aculeata (Hymenoptera: Chrysidoidea, Pompiloidea, Vespoidea, Ароidea) Шацкого национального природного парка (Волынская область, Украина). Известия Харьковского энтомологического общества. 2021. Т. ХХІХ, вып. 1. С. 8-19. DOI: 10.36016/KhESG-2021-29-1-2.

Приводится предварительный список Chrysididae, Mutillidae, Pompilidae, Vespidae, Sphecidae Crabronidae и Ароidea Шацкого национального природного парка. Среди 139 зарегистрированных в настоящее время 56 видов впервые указываются для территории парка, 5 видов занесены в Красные книги Украины, Беларуси и Польши, а 83 вида относятся к охраняемым категориям МСОП. Один инвазионный вид (Isodontia техісапа) впервые найден в Волынской области.

Ключевые слова: список видов, охранные категории, роющие осы, дорожные осы, пчёлы. 1 рис., 14 назв.

Kumpanenko, O. S., Honchar, H. Yu., Gorobchyshyn, V. A., Protsenko, Yu. V. Preliminary list of some Aculeata (Hymenoptera: Chrysidoidea, Pompiloidea, Vespoidea, Apoidea) of the Shatsk National Natural Park (Volyn Region, Ukraine). The Kharkov Entomological Society Gazette. 2021. Vol. XXIX, iss. 1. P. 8-19. DOI: 10.36016/KhESG-2021-29-1-2.

A preliminary checklist of Chrysididae, Mutillidae, Pompilidae, Vespidae, Sphecidae, Crabronidae, and Apoidea of the Shatsk National Natural Park are provided. Among 139 currently registered species, 56 species are recorded for the park territory for the first time, 5 species are listed in the Red Data Books of Ukraine, Belarus, and Poland, and 83 species belong to IUCN categories. One invasive species (Isodontia mexicana) was found in Volyn Region for the first time.

Keywords: checklist, red list, digger wasps, spider wasps, wild bees. 1 fig., 14 refs.

I n t rod u c t i o n. The Shatsk National Natural Park was established in 1983 at the territory of Volyn Region within the Ukrainian Polissya landscape zone. The park is located in the west of one of the largest swamp-lake-forest complexes of Europe. It was included in the World Network of Biosphere Reserves in 2002, and became the basis of the Ukrainian part of the International Polish-Belarusian-Ukrainian Biosphere Reserve 'Western Polissya' since 2011. The presence of eleven types of settlements NATURA 2000 in the park highlights its great role in various aspects of environmental conservation at the European level. The area of the Shatsk National Natural Park covers 48,977 hectares; its characteristic feature is the presence of a complex of lakes of various origins (there are 23 lakes in the park) with a total area of 6,338.9 hectares. Forests occupy 52.5\% of general park area, grasslands cover $12.4 \%$, marshes occupy $2.7 \%$, and water reservoirs cover $14.2 \%$. The rest of the area is taken by farms and roads (Yurchuk et al., 2014).

Thus, the study of biodiversity as a part of other conservation activities in the Shatsk National Natural Park is very actual. Hymenoptera, one of most taxonomically diverse order of insects, is studied in the park rather fragmentally. Thirty two species of wild bees were recorded by Osytshnjuk (1958, 1961, 1964), 25 species of bumblebees were given by Konovalova (2016), and 57 species of ants were registered by Radchenko (2009).

Kumpanenko O. S. Institute for Evolutionary Ecology of the National Academy of Sciences of Ukraine,

37, Lebedeva St., Kyiv, 03143, UKRAINE; e-mail: kumpanenko@gmail.com, ORCID: 0000-0003-4933-8654 Honchar H. Yu. Institute for Evolutionary Ecology of the National Academy of Sciences of Ukraine,

37, Lebedeva St., Kyiv, 03143, UKRAINE; e-mail: apantova@ukr.net, ORCID: 0000-0002-3429-5500 Gorobchyshyn V. A. Institute for Evolutionary Ecology of the National Academy of Sciences of Ukraine,

37, Lebedeva St., Kyiv, 03143, UKRAINE; e-mail: medziboz@yahoo.com, ORCID: 0000-0003-1896-5110 Protsenko Yu. V. Educational and Scientific Center 'Institute of Biology and Medicine', Taras Shevchenko National University of Kyiv, 2, Hlushkova Ave., Kyiv, 03127, UKRAINE; e-mail: yproc2012@gmail.com, ORCID: 0000-0001-9038-1182 
Materials and methods. A total of 579 specimens of different Aculeata were collected at 9-13 September 2019. Insects were caught with an entomological net and by colored pan traps (diameter - $75 \mathrm{~mm}$, height - $35 \mathrm{~mm}$, colors - white, yellow, and blue), which were placed in a transect of 15 traps. All sampling sites are shown on the map at Fig. 1. The species classification follows Wahis (2006), Ascher and Pickering (2020), Pulawski (2021). All identified specimens are deposited in the collection of the Institute for Evolutionary Ecology of the National Academy of Sciences of Ukraine (Kyiv, Ukraine).

Abbreviations used in the text: BY - Red Book of the Republic of Belarus (Kachanovskiy, Nikiforov, Parfenov, 2015); PL — Red Book of Animals of Polish (Głowaciński, Nowacki, 2004); UA — Red Data Book of Ukraine (Akimov, 2009). The categories of bees' species are also given by European Red List of Bees (Nieto et al., 2014): DD — data deficient, EN - endangered, LC - least concern, NT - near threatened, VU vulnerable.
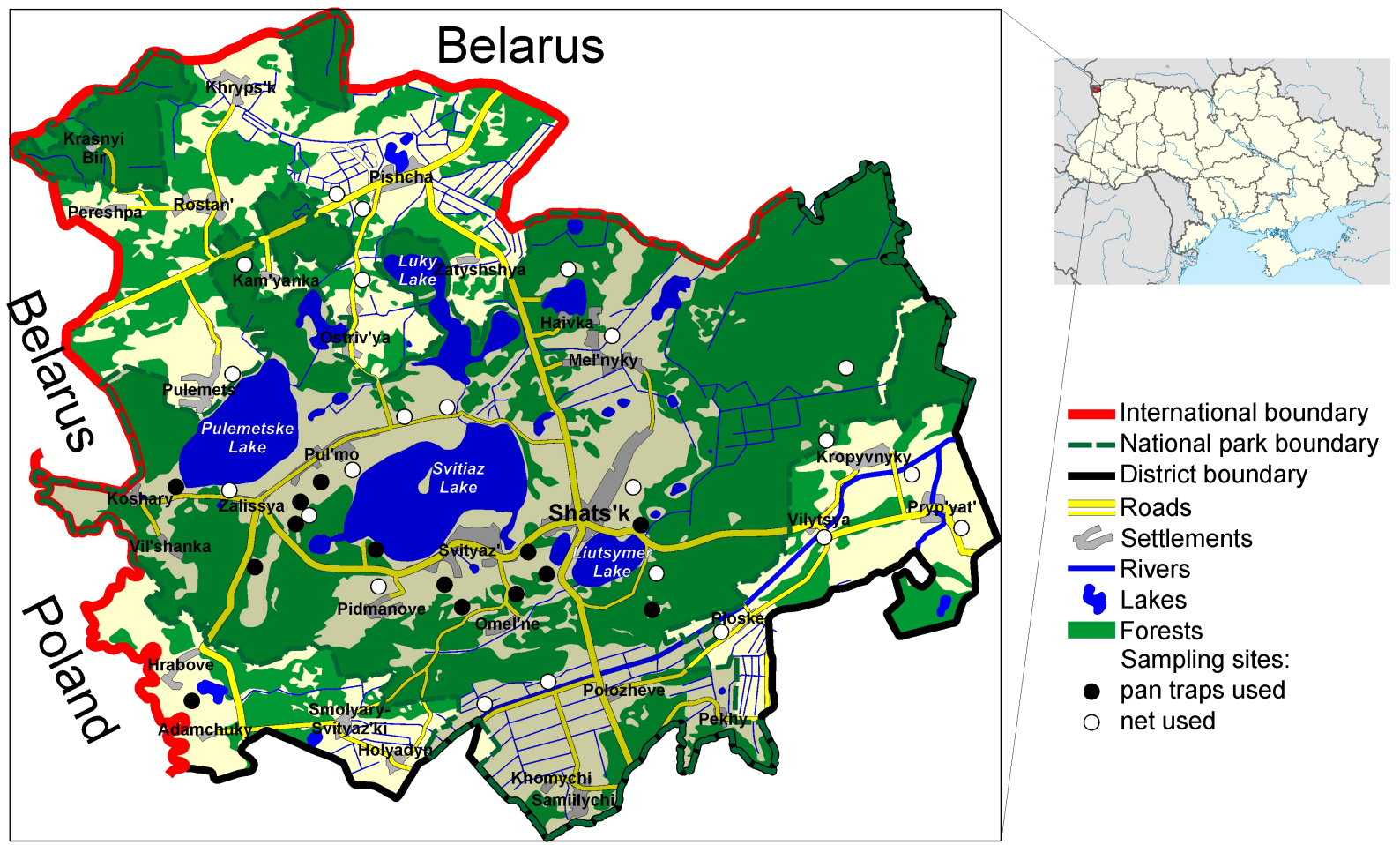

F i g . 1 . The map of the Shatsk National Natural Park with sampling sites.

Results a nd d iscussion. In the present contribution we provide the first results of our study of selected groups of Hymenoptera from the territory of the Shatsk National Natural Park. Thus, 56 species of Aculeata from the families Chrysididae, Mutillidae, Pompilidae, Vespidae, Sphecidae, and Crabronidae are recorded from the park for the first time. In addition, the list of the wild bees was supplemented by 29 new records. It should be noted that our survey took place in early autumn. Therefore, some phenological groups of insects (spring and early summer species) have not been registered.

Below we give the integrated checklist comprising 139 species currently known from the Shatsk National Natural Park. Species that were not found during our investigation, but are known from the literature are marked with '*'; species recorded from the park both from our study and known from the literature are marked with '**'.

Taking into account the specifics of this group of insects, we indicate the biotopic referencing, since the territory of the park is small.

F a m i l y C H R Y S I D I D A E Latreille, 1802

Subfamily CHRYS I In AE Latreille, 1802

Genus Chrysis Linnaeus, 1761

Chrysis bicolor (Lepeletier, 1805)

M a t e r i a l . Sandy area: $1 q-9 . I X .2019$ (pan trap). 
Chrysis splendidula Rossi, 1790

M a t e r i a l. Sandy area: 2 우 — 9.IX.2019 (pan trap), 1 \& - 11.IX.2019 (pan trap), 1 q — 12.IX.2019 (pan trap)

Genus Hedychrum Latreille, 1806

Hedychrum niemelai Linsenmaier, 1959

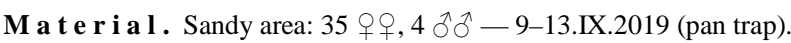

Family M U T I L L I D A L Latreille, 1802

Subfamily MUTILLINAE Latreille, 1802

Genus $S$ micromyrme Thomson, 1870

Smicromyrme (Smicromyrme) rufipes (Fabricius, 1787)

M a t e r i a l. Sandy area: $1+$ - 9.IX.2019 (net).

F a mily P O M P I L D A E Fabricius, 1798

S ubfamily PEPS I A E Lepeletier, 1845

Genus Caliadurgus Pate, 1946

Caliadurgus fas ciatellus (Spinola, 1808)

M a t e r i a l . Forest and forest edge: 2 우 — 9.IX.2019 (net - 1, pan trap - 1); grassland: 1 \& - 11.IX.2019 (net).

Genus Priocnemis Schioedte, 1837

Priocnemis (Priocnemis) exaltata (Fabricius, 1775)

M a t e r i a l . Forest and forest edge: 2 우 - 9.IX.2019 (pan trap).

Priocnemis (Priocnemis) fennica Haupt, 1927

M a t e r i a l. Forest and forest edge: 2 우 — 9.IX.2019 (pan trap).

S ubfamily P OMPILINAE Latreille, 1805

Genus Anoplius Dufour, 1834

Anoplius (Anoplius) concinnus (Dahlbom, 1843)

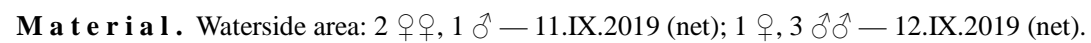

Anoplius (Anoplius) nigerrimus (Scopoli, 1763)

M a t e r i a l. Grassland: $1+-11 . I X .2019$ (net).

Anoplius (Arachnophroctonus) viaticus (Linnaeus, 1758)

M a t e r i a l. Forest and forest edge: 3 우 - 12.IX.2019 (net - 2, pan trap - 1).

Genus Arachnospila Kincaid, 1900

Arachnospila (Ammosphex) anceps (Wesmael, 1851)

M a t e r i a l. Grassland: $1+$ - 10.IX.2019 (net); sandy area: 2 우 — 9.IX.2019 (net).

Arachnospila (Ammosphex) trivialis (Dahlbom, 1843)

M a t e ri a l. Sandy area: 1 \& - 13.IX.2019 (pan trap).

Ge nu S Ep is yron Schioedte, 1837

Ep i syron albo notat um (Vander Linden, 1827)

M a t e r i a l. Grassland: 1 + — 10.IX.2019 (pan trap).

Episyron rufipes (Linnaeus, 1758)

M a t e r i a l. Sandy area: $1+$ - 9.IX.2019 (net), 3 $\circ$ 10.IX.2019 (net). 
Genus Evagetes Lepeletier, 1845

Evagetes (Evagetes) crassicornis (Shuckard, 1837)

M a t e r i a l. Grassland: 2 우 — 11.IX.2019 (net - 1, pan trap - 1); sandy area: 3 우 - 9.IX.2019 (net - 2, pan trap - 1), $1+-13 . I X .2019$ (net).

Evagetes (Evagetes) pectinipes (Linnaeus, 1758)

M a t e r i a l. Sandy area: 5 q $q$ - 10.IX.2019 (net), 1 \& - 12.IX.2019 (net).

Genus Pompilus Fabricius, 1798

Pompilus cinereus (Fabricius, 1775)

M a t e r i a l. Sandy area: 5 우 — 9.IX.2019 (net), $1+$ — 12.IX.2019 (net), 1 q, 1 ô — 13.IX.2019 (net).

F a mily VESP I A E Latreille, 1802

Subfamily EUMENINAE Leach, 1815

Genus Ancistrocerus Wesmael, 1836

Ancistrocerus gazella (Panzer, 1798)

M a t e r i a l. Grassland: 1 q - 10.IX.2019 (net).

Ancistrocerus trifas ciatus (Müller, 1776)

M a t e r i a l. Forest and forest edge: $1+$ - 9.IX.2019 (pan trap), $1+$ - 10.IX.2019 (net).

G e nus Euodynerus Dalla Torre, 1904

Euodynerus notatus (Jurine, 1807)

M a t e r i a l. Forest and forest edge: $1 q-12 . I X .2019$ (pan trap).

Ge nus Eumenes Latreille, 1802

Eumenes coarctatus (Linnaeus, 1758)

M a t e ri a l. Forest and forest edge: 2 ㅇ - 9.IX.2019 (net - 1, pan trap - 1); grassland: 2 우 - 11.IX.2019 (pan trap), 1 ㅇ - 13.IX.2019 (net); sandy area: 2 우 — 9.IX.2019 (pan trap), 2 우 — 13.IX.2019 (net).

Eumenes coronatus (Panzer, 1799)

M a t e ri a l. Forest and forest edge: $1+$ - 9.IX.2019 (pan trap).

Eumenes pedunculatus (Panzer, 1799)

M a t e r i a l. Forest and forest edge: $1+-11 . I X .2019$ (net).

Genus Stenodynerus Saussure, 1863

Stenodynerus orenburgensis (André, 1884)

M a t e r i a l. Grassland: 1 đ - 11.IX.2019 (pan trap).

Subfamily Polis TINAE Bequaert, 1918

Genus Polistes (Latreille, 1802)

Polistes (Polistes) gallicus (Linnaeus, 1767) ${ }^{\mathrm{PL}}$

M a t e r i a l. Grassland: 1 , 1 o — 12.IX.2019 (net).

Polistes (Polistes) nimpha (Christ, 1791)

M a t e r i a l. Grassland: 1 worker - 10.IX.2019 (pan trap).

Subfamily VESP INAE Latreille, 1802

G e nus Ve s pa Linnaeus, 1758

Vespa crabro Linnaeus, 1758

M a t e r i a l. Forest and forest edge: 1 worker - 9.IX.2019 (pan trap). 
Family SPHECIDAE Latreille, 1802

S ubfamily Am о P hil in A E André, 1886

Genus Ammophila W. Kirby, 1798

Ammophila campestris Latreille, 1809

M a t e r i a l. Grassland: 2 우 — 10.IX.2019 (pan trap), 3 우 — 13.IX.2019 (pan trap); sandy area: 4 우 — 11.IX.2019 (pan trap), 1 q - 12.IX.2019 (net).

Ammophila sabulosa (Linnaeus, 1758)

M a t e r i a l. Grassland: 4 우 — 10.IX.2019 (pan trap), 2 우 — 12.IX.2019 (pan trap); sandy area: 1 13.IX.2019 (net).

Genus Podalonia Fernald, 1927

Podalonia affinis (W. Kirby, 1798)

M a t e r i a l. Grassland: 10 우 — 8-13.IX.2019 (pan trap); sandy area: 1 + — 13.IX.2019 (net).

Subfamily SPHEC I A E Latreille, 1802

Genus Is odontia (Patton, 1880)

Isodontia mexicana (Saussure, 1867)

M a t e r i a l. Grassland: $1 q-12 . I X .2019$ (pan trap).

Ge nus Prionyx Vander Linden, 1827

Prionyx nudatus (Kohl, 1885)

M a t e $\mathbf{r}$ i a l. Grassland: 3 우 — 12.IX.2019 (pan trap); sandy area: 1 + — 12.IX.2019 (net).

Genus $S$ phex Linnaeus, 1758

Sphex funerarius Gussakovskij, $1934^{\text {BY, UA }}$

M a t e r i a l. Grassland: 3 우 — 12.IX.2019 (pan trap).

Family CRABRON IDAE Latreille, 1802

S u bf a mily B EM B I C I N A E Latreille, 1802

Genus Didine is Wesmael, 1852

Didine is Iunicornis (Fabricius, 1798)

M a t e r i a l. Grassland: 1 - $-12 . I X .2019$ (net).

Genus Gorytes Latreille, 1804

Gorytes quinquecinctus (Fabricius, 1793)

M a t e ri a l. Forest and forest edge: 4 우우 - 13.IX.2019 (net).

Genus Harpactus Shuckard, 1837

Harpactus morawitzi Radoszkowski, 1884

M a t e r i a l. Grassland: 1 - $-12 . I X .2019$ (net).

Subfamily CRABRONINAE Latreille, 1802

Genus Crabro Fabricius, 1775

Crabro scutellatus (Scheven, 1781)

M a t e r i a l . Forest and forest edge: 1 §-11.IX.2019 (net).

G e nus Cros s o c e rus Lepeletier et Brullé, 1835

Crossocerus (Blepharipus) annulipes (Lepeletier et Brullé, 1835)

M a t e ri a l. Forest and forest edge: 2 우 $-13.1 X .2019$ (net). 
Genus Ectemnius Dahlbom, 1845

Ectemnius (Hypocrabro) continuus (Fabricius, 1804)

M a t e r i a l. Grassland: $1+-10 . I X .2019$ (pan trap).

Ge nus Le stica Billberg, 1820

Lestica clypeata (Linnaeus, 1767)

M a t e r i a l. Grassland: 2 우, 1 §ิ-10.IX.2019 (pan trap), 2 우 — 12.IX.2019 (pan trap).

G e nus Lindenius Lepeletier et Brullé, 1835

Lindenius albilabris (Fabricius, 1793)

M a t e r i a l. Grassland: 1 - — 10.IX.2019 (pan trap).

Genus Oxybelus Latreille, 1796

Oxybelus quatuordecimnotatus Jurine, 1807

M a t e r i a l. Grassland: 1 , 1 $\precsim-10 . I X .2019$ (pan trap).

Oxybelus trispinosus (Fabricius, 1787)

M a t e ri a l. Grassland: $1 q-12$. IX.2019 (pan trap).

Genus Tachysphex Kohl, 1883

Tachysphex nitidior Beaumont, 1940

M a t e r i a l. Sandy area: 2 우 - 9.IX.2019 (net).

Genus Trypoxylon Latreille, 1796

Trypoxylon (Trypoxylon) attenuatum Smith, 1851

M a t e r i a l. Grassland: 6 우 — 9-13.IX.2019 (pan trap).

Subfamily MELLININAE Latreille, 1802

Genus Mellinus Fabricius, 1790

Mellinus arvensis (Linnaeus, 1758)

M a t e r i a l. Forest and forest edge: $2 \uparrow q, 1 \hat{\jmath}-9 . I X .2019$ (net); grassland: 15 $\uparrow+$ - 9-13.IX.2019 (pan trap); sandy area: 2 우우 - 13.IX.2019 (net).

Subfamily PEMPHRED O I N A Dahlbom, 1835

Genus Diodontus Curtis, 1834

Diodontus minutus (Fabricius, 1793)

M a t e r i a l. Grassland: 2 우, $1 \hat{\jmath}-12 . I X .2019$ (pan trap).

Genus Pemphredon Latreille, 1796

Pemphredon (Cemonus) inornata Say, 1824

M a t e r i a l . Forest and forest edge: $1+$ - 13.IX.2019 (net).

Pemphredon (Pemphredon) montana Dahlbom, 1845

M a t e r i a l. Grassland: 1 - $-12 . I X .2019$ (pan trap).

Subfamily PHiLA N T I N A E Latreille, 1802

Genus Cerceris Latreille, 1802

Cerceris arenaria (Linnaeus, 1758)

M a t e r i a l. Grassland: 16 우, $5 \hat{\jmath}$ - -9 -13.IX.2019 (pan trap); sandy area: 1 q — 10.IX.2019 (net), 2 우 — 13.IX.2019 (net - 1, pan trap - 2).

Cerceris interrupta (Panzer, 1799)

M a t e r i a l. Grassland: $1+-10 . I X .2019$ (pan trap). 
Cerceris quadricincta (Villers, 1799)

M a t e ri a l . Grassland: $1 \hat{\delta}-12 . I X .2019$ (pan trap).

Cerceris quinquefasciata (Rossi, 1792)

M a t e r i a l. Grassland: $1 q-11 . I X .2019$ (pan trap).

Cerceris rybyensis (Linnaeus, 1771)

M a t e ri a l . Grassland: 5 $q$ - 9-13.IX.2019 (pan trap).

Cerceris sabulosa (Panzer, 1799)

M a t e r i a l. Grassland: 10 우, 1 ○ - 9-13.IX.2019 (pan trap).

Genus Philanthus Fabricius, 1790

Philanthus triangulum (Fabricius, 1775)

M a t e r i a l. Grassland: 2 우, 1 §ิ-12.IX.2019 (net).

F a mi ly C O L L E T I D A E Lepeletier, 1841

S ubfamily C OLLETINAE Lepeletier, 1841

Genus Colletes Latreille, 1802

** Colletes (Colletes) succinctus (Linnaeus, 1758) ${ }^{\mathrm{NT}}$

M a t e r i a l . Forest, dry heaths, forest edge: 2 우 — 12.IX.2019 (net), 1 § — 10.IX.2019 (net).

* Colletes (Simcolletes) fodiens (Geoffroy, 1785) ${ }^{\mathrm{vU}}$

Colletes (Simcolletes) similis Schenck, $1853^{\mathrm{LC}}$

M a t e r i a l. Grassland: 2 우 — 10.IX.2019 (net)

S ubfamily H Y L A I NAE Viereck, 1916

Genus Hyla eus Fabricius, 1793

Hylaeus (Abrupta) cornutus Curtis, $1831^{\text {LC }}$

M a t e r i a l. Grassland: 2 q $q-10 . I X .2019$ (pan trap).

Hylaeus (Dentigera) brevicornis Nylander, $1852^{\mathrm{LC}}$

M a t e r i a l. Grassland: 1 우 - 09.IX.2019 (pan trap).

*Hylaeus (Hylaeus) angustatus (Schenck, 1861) ${ }^{\mathrm{LC}}$

Hylaeus (Hylaeus) annularis (Kirby, 1802) ${ }^{\text {DD }}$

M a t e r i a l. Grassland: 1 - 10.IX.2019 (net).

Hylaeus (Hylaeus) communis Nylander, $1852^{\mathrm{LC}}$

M a t e r i a l. Grassland: 1 ô-12.IX.2019 (net).

Family ANDRENIDAE Latreille, 1802

Subfamily AndreninAE Latreille, 1802

Genus Andrena Fabricius, 1775

* Andrena (Cnemidandrena) denticulata (Kirby, 1802) ${ }^{\text {DD }}$

Andrena (Cnemidandrena) fuscipes (Kirby, 1802) ${ }^{\mathrm{DD}}$

M a t e r i a l. Forest, dry heaths: $1+$ - 11.IX.2019 (net).

* Andrena (Hoplandrena) rosae Panzer, $1801^{\mathrm{DD}}$

* Andrena (Melandrena) gallica Schmiedeknecht, $1883^{\mathrm{NT}}$ 
Subfamily PANURGINAE Leach, 1815

Genus Panurginus Nylander, 1848

Panurginus labiatus Eversmann, $1852^{\mathrm{DD}}$

M a t e r i a l. Grassland: 1 \& - 12.IX.2019 (pan trap).

F amily HALICT I A E Thomson, 1869

Subfamily HALICTINAE Thomson, 1869

Genus Halictus Latreille, 1804

*Halictus (Halictus) quadricinctus (Fabricius, 1776) ${ }^{\text {NT }}$

Halictus (Protohalictus) rubicundus (Christ, 1791) ${ }^{\text {LC }}$

M a t e r i a l. Grassland: 1 , 1 ô-10.IX.2019 (net).

Halictus (Seladonia) subauratus (Rossi, 1792) ${ }^{\text {LC }}$

M a t e r i a l. Grassland: $1 q-10.1 X .2019$ (net), $1 q-11 . I X .2019$ (net); sandy area: 2 + $q$ - 11.IX.2019 (net), $3 \hat{\jmath} \hat{o}-$ 12.IX.2019 (pan trap).

Halictus (Seladonia) tumulorum (Linnaeus, 1758) ${ }^{\mathrm{LC}}$ trap - 8).

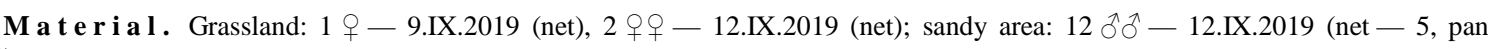

Genus Lasiogloss um Curtis, 1833

Lasioglossum (Dialictus) leucopus (Kirby, 1802) ${ }^{\mathrm{LC}}$

M a t e r i a l. Grassland: 1 ô-9.IX.2019 (net).

Lasioglossum (Hemihalictus) lucidulum (Schenk, 1861) ${ }^{\text {LC }}$

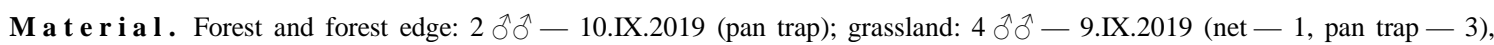

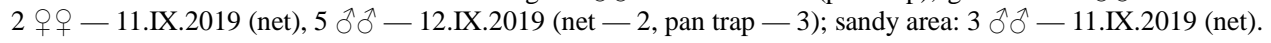

** Lasioglossum (Hemihalictus) minutissimum (Kirby, 1802) ${ }^{\text {LC }}$

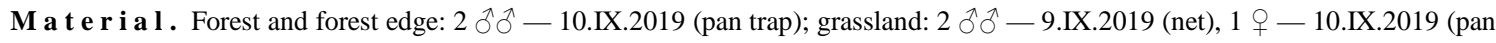

trap).

*Lasioglossum (Hemihalictus) punctatissimum (Schenck, 1853) ${ }^{\text {LC }}$

*Lasioglossum (Hemihalictus) quadrinotatulum (Schenck, 1861) ${ }^{\mathrm{NT}}$

*Lasioglossum (Hemihalictus) semilucens (Alfken, 1914) ${ }^{\text {LC }}$

* Lasioglossum (Hemihalictus) tarsatum (Schenck, 1869) ${ }^{\mathrm{NT}}$

* Lasioglossum (Lasioglossum) sexnotatum (Kirby, 1802) ${ }^{\mathrm{NT}}$

Lasioglossum (Lasioglossum) discum (Smith 1853) ${ }^{\text {LC }}$

M a t e r i a l. Sandy area: $1+$ - 12.IX.2019 (net).

Lasioglossum (Lasioglossum) pallens (Brullé, 1832) ${ }^{\text {LC }}$

M a t e ri a l. Sandy area: $1+-13 . I X .2019$ (net).

* Lasioglossum (Lasioglossum) quadrinotatum (Kirby, 1802) ${ }^{\mathrm{NT}}$

Lasioglossum (Leuchalictus) leucozonium (Schrank, 1781) ${ }^{\text {LC }}$

M a t e r i a l. Grassland: 1 \& - 10.IX.2019 (pan trap), $2 \widehat{\jmath} \widehat{o}-13 . I X .2019$ (net).

Lasioglossum (Leuchalictus) zonulum (F. Smith, 1848) ${ }^{\text {LC }}$

M a t e r i a l. Grassland: 1 \& 10.IX.2019 (net); sandy area: 1 \& - 13.IX.2019 (net).

Lasioglossum (Sphecodogastra) albipes (Fabricius 1781) ${ }^{\text {LC }}$

M a t e ri a l. Sandy area: 1 \& - 11.IX.2019 (pan trap).

** Lasioglossum (Sphecodogastra) calceatum (Scopoli, 1763) ${ }^{\text {LC }}$

M a t e r i a l. Sandy area: $2 \widehat{\jmath} \delta^{-}-09 . I X .2019$ (net), 1 + — 10.IX.2019 (net). 
* Lasioglossum (Sphecodogastra) laeve (Kirby, 1802) ${ }^{\mathrm{EN}}$

Lasioglossum (Sphecodogastra) malachurum (Kirby, 1802) ${ }^{\text {LC }}$

M a t e r i a l. Sandy area: 1 \& — 10.IX.2019 (net), 1 § - 13.IX.2019 (pan trap).

Genus Sphecodes Latreille, 1804

** Sphecodes monilicornis (Kirby, 1802) ${ }^{\text {LC }}$

M a t e r i a l. Sandy area: 1 \& - 11.IX.2019 (net), $1 \widehat{\delta}$ — 12.IX.2019 (net).

Sphecodes puncticeps Thomson, $1870^{\mathrm{LC}}$

M a t e r i a l. Sandy area: 1 , $1 \hat{\jmath}-10 . I X .2019$ (net), $1 \hat{\delta}-12 . I X .2019$ (pan).

* Sphecodes reticulatus Thomson, $1870^{\mathrm{LC}}$

* Sphecodes rubicundus Hagens, $1875^{\mathrm{NT}}$

F amily M E L I T T I D A E Michener, 2000

S ubfamily D A S Y P O D I N A E Börner, 1919

Gen us Dasypoda Latreille, 1802

Dasypoda (Dasypoda) hirtipes (Fabricius, 1793) ${ }^{\text {LC }}$

M a t e r i a l. Grassland: $1 q-12 . I X .2019$ (net); sandy area: $1 q-$ 09.IX.2019 (pan trap), $1+$ - 10.IX.2019 (pan trap).

Subfamily MaCROPID In AE Robertson, 1904

Genus Macropis Panzer, 1809

* Macropis (Macropis) europaea Warncke, $1973^{\mathrm{LC}}$

S ubfamily MEL I T T I N A E Schenk, 1861

Genus Melitta Kirby, 1802

* Melitta (Cilissa) haemorrhoidalis (Fabricius, 1775) ${ }^{\text {LC }}$

* Melitta (Melitta) nigricans Alfken, $1905^{\mathrm{LC}}$

Melitta (Melitta) tricincta Kirby, $1802^{\mathrm{NT}}$

M a t e r i a l. Grassland: $4+q, 10 \uparrow-13 . I X .2019$ (net).

F a mily M E G A C H I L I A E Latreille, 1802

Subfamily MEgACHIL INAE Latreille, 1802

Genus Anthidiellum Cockerell, 1904

*Anthidiellum (Anthidiellum) strigatum (Panzer, 1805) ${ }^{\text {LC }}$

Genus Anthidium Fabricius, 1805

Anthidium (Anthidium) manicatum (Linnaeus, 1758) ${ }^{\text {LC }}$

M a t e r i a l. Grassland: 1 کै-11.IX.2019 (net).

Anthidium (Anthidium) septemspinosum Lepeletier, 1841 DD

M a t e r i a l. Grassland: $1 \hat{\jmath}-11$. IX.2019 (net).

Genus Che los toma Latreille, 1809

*Chelostoma (Chelostoma) florisomne (Linnaeus, 1758) ${ }^{\mathrm{LC}}$

Ge nus Coe lioxys Latreille, 1809

Coelioxys (Boreocoelioxys) mandibularis Nylander, $1848^{\text {LC }}$

M a t e r i a l. Sandy area: 1 , $1 \hat{o}-13 . I X .2019$ (net). 
Ge nus Megachile Latreille, 1802

* Megachile (Megachile) alpicola Alfken, $1924^{\text {DD }}$

Megachile (Megachile) Iigniseca (Kirby, 1802) ${ }^{\mathrm{DD}}$

M a t e ri a l. Grassland: 1 - $-11.1 X .2019$ (net).

Megachile (Megachile) versicolor Smith, $1844^{\text {DD }}$

M a t e r i a l. Forest and forest edge: 5 + $q-10 . I X .2019$ (net - 2, pan trap - 3); grassland: 7 + $q-9 . I X .2019$ (net -3 , pan trap - 4); sandy area: 6 우, $1 \hat{\jmath}-13 . I X .2019$ (net -1 , pan trap - 1).

Family A P I A E Latreille, 1802

Subfamily APInAE Latreille, 1802

Genus Anthophora Latreille, 1803

**Anthophora (Clisodon) furcata (Panzer, 1798) ${ }^{\mathrm{LC}}$

M a t e r i a l. Grassland: 3 우 — 11.IX.2019 (net).

G e nus B ombus Latreille, 1802

* Bombus (Bombias) confusus Schenck, $1859^{\mathrm{VU}}$

** Bombus (Bombus) I ucorum (Linnaeus, 1761) ${ }^{\text {LC }}$

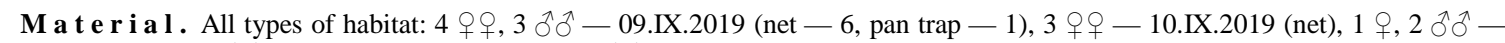

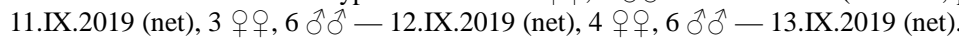

** Bombus (Bombus) terrestris (Linnaeus, 1758) ${ }^{\text {LC }}$

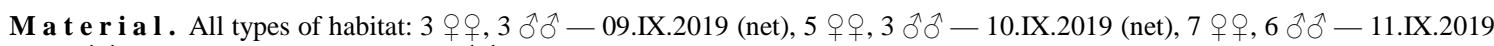

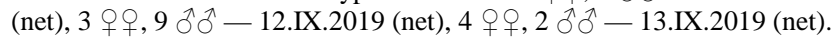

* Bombus (Cullumanobombus) semenoviellus Scorikov, $1910^{\mathrm{LC}}$

* Bombus (Kallobombus) soroeensis (Fabricius, 1776) ${ }^{\mathrm{LC}}$

** Bombus (Megabombus) hortorum (Linnaeus, 1761) ${ }^{\text {LC }}$

M a t e r i a l. All types of habitat: 3 우, $4 \hat{\delta} \hat{\sigma}-09 . I X .2019$ (net - 5, pan trap - 2), 2 우 — 10.IX.2019 (net), 4 우, $2 \hat{\delta} \hat{\delta}-$ 11.IX.2019 (net), 2 우, 3 o $^{\lambda}-12 . I X .2019$ (net), 1 + - 13.IX.2019 (pan trap).

Bombus (Megabombus) ruderatus (Fabricius, 1775) ${ }^{\text {LC }}$

M a t e $\mathbf{r}$ i a l. Grassland: 2 우, 1 ô — 09.IX.2019 (net), 1 ㅇ - 11.IX.2019 (net), 1 ô - 13.IX.2019 (net).

* Bombus (Melanobombus) lapidarius (Linnaeus, 1758) ${ }^{\text {LC }}$

* Bombus (Psithyrus) barbutellus (Kirby, 1802) ${ }^{\mathrm{LC}}$

* Bombus (Psithyrus) bohemicus Seidl, $1837^{\text {LC }}$

** Bombus (Psithyrus) campestris (Panzer, 1801) ${ }^{\text {LC }}$

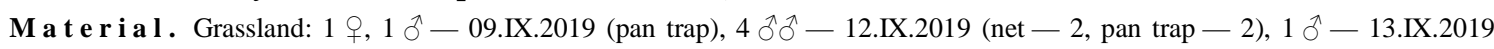
(net).

* Bombus (Psithyrus) norvegicus (Sparre-Schneider, 1918) ${ }^{\text {LC }}$

**Bombus (Psithyrus) rupestris (Fabricius, 1793) ${ }^{\text {LC }}$

M a t e ri a l. Grassland: $1 \hat{o}-11 . I X .2019$ (pan trap).

** Bombus (Psithyrus) sylvestris (Lepeletier, 1832) ${ }^{\text {LC }}$

M a t e r i a l. Grassland: 1 - 09.IX.2019 (pan trap), 2 우 — 12.IX.2019 (net — 1, pan trap - 1).

*Bombus (Psithyrus) vestalis (Geoffroy, 1785) ${ }^{\mathrm{LC}}$

* Bombus (Pyrobombus) hypnorum (Linnaeus, 1758) ${ }^{\text {LC }}$

**Bombus (Pyrobombus) jonellus (Kirby, 1802) ${ }^{\text {LC }}$

M a t e r i a l. Forest and forest edge: $1 \hat{\jmath}-11$. IX.2019 (net).

* Bombus (Pyrobombus) pratorum (Linnaeus, 1761) ${ }^{\text {LC }}$ 
* Bombus ( Subterraneobombus) distinguendus Morawitz, 1869 VU, BY

* Bombus ( Subterraneobombus) subterraneus (Linnaeus, 1758) ${ }^{\text {LC }}$

Bombus ( Thoracobombus) humilis Illiger, $1806^{\mathrm{LC}}$

M a t e r i a l. Forest and forest edge: 1 ^ — 11.IX.2019 (net); grassland: 1 ㅇ — 09.IX.2019 (net).

** B ombus (Thoracobombus) muscorum (Linnaeus, 1758) VU, BY, UA

M a t e r i a l. Grassland: 2 workers - 12.IX.2019 (pan trap).

** Bombus (Thoracobombus) pascuorum (Scopoli, 1763) ${ }^{\text {LC }}$

M a t e r i a l. All types of habitat: 10 우, $12 \hat{\jmath} \widehat{\delta}-09 . I X .2019$ (net - 19, pan trap - 3), 12 우, $9 \hat{\jmath} \widehat{\delta}-10 . \mathrm{IX} .2019$ (net -

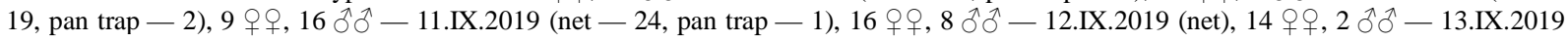
(net - 14, pan trap - 2).

** B ombus ( Thoracobombus) ruderarius (Müller, 1776) ${ }^{\text {LC }}$

M a t e r i a l . Forest and forest edge: $1 \hat{\jmath}-13 . I X .2019$ (net); grassland: $1 \hat{\jmath}-10.1 X .2019$ (net).

* Bombus ( Thoracobombus) schrencki Morawitz, $1881^{\text {LC, BY }}$

** Bombus ( Thoracobombus) sylvarum (Linnaeus, 1761) ${ }^{\text {LC }}$ trap - 1).

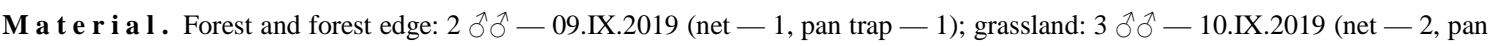

** Bombus (Thoracobombus) veteranus (Fabricius, 1793) ${ }^{\text {LC }}$

M a t e r i a l . Grassland: $1 \hat{\delta}-10 . I X .2019$ (net).

G e nus Eucera Scopoli, 1770

* Eucera malvae (Rossi, 1790) ${ }^{\text {LC }}$

S u b f a m i l y N O M D I N E Latreille, 1802

Ge nus Epeo I us Latreille, 1802

** Epeolus cruciger (Panzer, 1799) ${ }^{\mathrm{NT}}$

M a t e r i a l. Sandy area: 1 - -13. IX.2019 (net).

Epeolus variegatus (Linnaeus, 1758) ${ }^{\text {LC }}$

M a t e r i a l . Sandy area: 1 - -12 .IX.2019 (net).

Ge nus Nom ad a Scopoli, 1770

* Nomada fuscicorn is Nylander, $1848^{\text {LC }}$

This list comprises 139 species from twelve families of Aculeata. Among them 20 species (including seven species known only from the literature) are kleptoparasites from the genera Chrysis, Hedychrum, Evagetes, Sphecodes, Coelioxys, Epeolus, Nomada and the subgenus Psithyrus. Isodontia mexicana, an invasive species from North America, is recorded for this region for the first time.

About $40 \%$ of the wild bees are specialized in collecting pollen from certain plant species. The specialized species recorded at the territory of the park are: for flowers of Calluna vulgaris (L.) Hull (Ericaceae) — Andrena fuscipes, Colletes succinctus, for Odontites vulgaris Moench (Orobanchaceae) - Melitta tricincta, and for Campanula spp. (Campanulaceae) - Melitta haemorrhoidalis. In addition, some wild bees collect pollen from certain families: Asteraceae - Andrena denticulata, Colletes fodiens, Colletes similis, Dasypoda hirtipes, Heriades truncorum, Megachile ligniseca; Brassicaceae — Panurginus labiatus; Campanulaceae — Chelostoma florisomne; Lamiaceae - Anthophora furcata; Lythraceae - Melitta nigricans; Malvaceae - Tetralonia malvae; Primulaceae - Macropis europaea (Radchenko, Pesenko, 1994).

Conclus i on s. A total of 139 species from 12 families of Aculeata are currently registered from the territory of the Shatsk National Natural Park. Some species of the families Chrysididae, Mutillidae, Pompilidae, Vespidae, Sphecidae, and Crabronidae are recorded for the first time.

The list also comprises five species protected by the Red Data Books of Ukraine, Belarus, and Poland: Bombus distinguendus (BY), Bombus muscorum (BY, UA), Bombus schrencki (BY), Polistes gallicus (PL), Sphex funerarius (BY, UA). Among the registered 83 species, which have the IUCN categories, are those belonging to: EN - 1 species, VU - 4 species, NT - 10 species, and DD - 9 species. There are wild bees, 
which are specialized to collect pollen from certain families (12 species) or only some species of flowers (4 species) too. The occurrence of rare species, and those whose population status cannot be estimated, indicates the importance of the Shatsk National Natural Park for the protection of insect diversity. In addition, one invasive species, Isodontia mexicana, is recorded for Volyn Region for the first time.

Acknowledgements. This work was funded by the State Budget Program 'Support for the Development of Priority Areas of Scientific Research’ (Code: 6541230). The authors thank to Sam Droege (Patuxent Wildlife Research Center, Upper Marlboro, Maryland, USA) for the organization expedition and help in the field work, Kateryna Martynova (I. I. Schmalhausen Institute of Zoology of National Academy of Sciences of Ukraine, Kyiv, Ukraine) - for the identification of Chrysididae species, and Oleksiy Bidzilya (Institute for Evolutionary Ecology of the National Academy of Sciences of Ukraine, Kyiv, Ukraine) for corrections and constructive comments to the text.

\section{R E F E R E N C E S}

Akimov, I. A., ed. 2009. Red Data Book of Ukraine. Animals [Червона книга України. Тваринний світ]. Hlobalkonsaltynh, Kyiv, 1-624. ISBN: 9789669705907. [in Ukrainian].

Ascher, J. S., Pickering, J. 2020. Discover Life bee species guide and world checklist (Hymenoptera: Apoidea: Anthophila). Draft-55, 17 November, 2020. URL: https://www.discoverlife.org/mp/20q?guide=Apoidea_species.

Glowaciński, Z., Nowacki, J., eds. 2004. The Red Book of Animals of Polish: Invertebrates [Polska czerwona księga zwierzat. Bezkręgowce]. Instytut Ochrony Przyrody PAN, Akademia Rolnicza im. A. Cieszkowskiego, Kraków; Poznań, 1-447. ISBN: 8388934600 .

Kachanovskiy, I. M., Nikiforov, M. E., Parfenov, V. I., eds. 2015. The Red Book of the Republic of Belarus. Animals. Rare and endangered species of wild animals [Красная книга Республики Беларусь. Животные. Редкие и находящиеся под угрозой исчезновения виды диких животных]. $4^{\text {th }}$ ed. Belaruskaya Entsyklapedyya, Minsk, 1-317. ISBN: 9789851108448. [in Russian]

Konovalova, I. B. 2016. Bumblebees - Bombus Latreille, 1802 [Джмелі - Bombus Latreille, 1802]. In: Kilochytskyi, P. Ya., ed. Shatsk Lakes. Volume 8. Fauna [Шацьке Поозер'я. Том 8. Тваринний світ]. CD-ROM ed. Vezha-Druk, Lutsk, 329-337. ISBN: 9789669400376. URL: http://zoomus.lviv.ua/files/monograf biolog\%202016.pdf. [in Ukrainian].

Nieto, A., Roberts, S. P. M., Kemp, J., Rasmont, P., Kuhlmann, M., García Criado, M., Biesmeijer, J. C., Bogusch, P., Dathe, H. H., Dela Rúa, P., De Meulemeester, T., Dehon, M., Dewulf, A., Ortiz-Sánchez, F. J., Lhomme, P., Pauly, A., Potts, S. G., Praz, C., Quaranta, M., Radchenko, V. G., Scheuchl, E., Smit, J., Straka, J., Terzo, M., Tomozii, B., Window, J., Michez, D. 2014. European Red List of Bees. Publication Office of the European Union, Luxembourg. i-x, 1-86. DOI: https://doi.org/10.2779/77003.

Osytshnjuk, A. Z. 1958. Some data on bees of the Volyn Region. [Некоторые данные по пчёлам Волынской области]. In: $1^{\text {st }}$ Zoological Conference of the Byelorussian SSR: Abstracts [Первая зоологическая конференция Белорусской ССР: тезисы докладов]. Minsk, 183-184. [in Russian].

Osytshnjuk, A. Z. 1961. To study the fauna and ecology of bees of Western Polissya of Ukraine [До вивчення фауни і екологіi бджолиних Західного Полісся України]. Proceedings of the Institute of Zoology of the Academy of Sciences of the Ukrainian SSR [Прaui Інституту зоології АН УРСР], 17, 99-107. [in Ukrainian].

Osytshnjuk, A. Z. 1964. The bees (Apoidea) of the Ukrainian Polissya [Бджолині (Ароidea) Українського Полісся]. Proceеdings of the Institute of Zoology of the Academy of Sciences of the Ukrainian SSR [Праиі Iнституту зоологіï АН УРCP], 20, 120-149. [in Ukrainian].

Pulawski, W. J. 2021. Catalog of Sphecidae sensu lato (= Apoidea excluding Apidae). URL: https://www.calacademy.org/scientists/projects/ catalog-of-sphecidae.

Radchenko, O. G. 2009. The fauna, zoogeographical peculiarities and the provision of ant protection in Shatsk National Natural Park. [Фауна, зоогеографічні особливості та необхідність охорони мурашок Шацького національного природного парку]. Lеsia Ukrainka Volyn National University Scientific Bulletin. Series: Biological Sciences [Науковий вісник Волинського національного університету імені Лесі Украӥнки. Серія: Біологічні науки], 2, 149-153. URL: http://nbuv.gov.ua/UJRN/Nvvnu_2009_2_29. [in Ukrainian].

Radchenko, V. G., Pesenko, Yu. A. 1994. Biology of Bees (Hymenoptera, Apoidea) [Биология пчел (Нутепорtеra, Apoidea)]. Zoological Institute of the Russian Academy of Sciences, Saint Petersburg: 1-350. [in Russian].

Wahis, R. 2006. Mise à jour du Catalogue systématique des Hyménoptères Pompilides de la région ouesteuropéenne. Additions et Corrections. Notes Fauniques de Gembloux, 59(1), 31-36.

Yurchuk, P. V., Mateichyk, V. I., Yashchenko, P. T., Shydlovskyi, I. V., Horban, I. M., Pisulinska, N. A., eds. 2014. Rarities of Biota of the Shatsk National Natural Park (distribution, habitat, threats, and conservation) [Раритети біоти Шацького національного природного парку (поширення, оселища, загрози та збереження)]. Komprynt, Kyiv, 1-219. ISBN: 9786177144846. [in Ukrainian].

Institute for Evolutionary Ecology of the National Academy of Sciences of Ukraine

Taras Shevchenko National University of Kyiv 